\title{
Single-incision laparoscopic total gastrectomy with D1+beta lymph node dissection for proximal early gastric cancer
}

\author{
Sang-Hoon Ahn · Do Joong Park · Sang-Yong Son • \\ Chang-Min Lee $\cdot$ Hyung-Ho Kim
}

Received: 28 February 2013/ Accepted: 29 April 2013/Published online: 17 May 2013

(c) The International Gastric Cancer Association and The Japanese Gastric Cancer Association 2013

\begin{abstract}
Single-incision laparoscopic distal gastrectomy for early gastric cancer has recently been reported by a few centers in Korea and Japan. In this technical report, we describe the world's first pure single-incision laparoscopic total gastrectomy with $\mathrm{D} 1+$ beta lymph node dissection for proximal early gastric cancer.
\end{abstract}

Keywords Single-incision laparoscopic surgery (SILS) · Total gastrectomy $\cdot$ Single-incision laparoscopic total gastrectomy (SITG) · Gastric cancer · Lymph node dissection

\section{Introduction}

As the result of early detection of gastric cancer and technological advances, minimally invasive surgery (MIS) has become the main axis of treatment. In particular, laparoscopic gastrectomy for EGC has been widely accepted as an alternative to open gastrectomy in Korea and Japan $[1,2]$. Further, new emerging techniques have been developed to reduce the invasiveness of laparoscopic surgery, such as the natural orifice transluminal endoscopic surgery (NOTES) and single-incision laparoscopic surgery (SILS). Although NOTES is still being studied because of the limitations of current instruments, SILS has been

S.-H. Ahn · D. J. Park · S.-Y. Son · C.-M. Lee · H.-H. Kim Department of Surgery, Seoul National University College of Medicine, Seoul, Korea

S.-H. Ahn · D. J. Park · S.-Y. Son · C.-M. Lee · H.-H. Kim ( $\square)$ Department of Surgery, Seoul National Bundang Hospital, 300 Gumi-dong, Bundang-gu,

Seongnam, Gyenggi 463-707, Korea

e-mail: hhkim@snubh.org frequently applied in various clinical settings, such as cholecystectomy, appendectomy, colectomy, and sleeve gastrectomy for obesity [3, 4]. However, there have been only three reports about single-incision laparoscopic distal gastrectomy (SIDG) for gastric cancers [5-7]. Herein, we report a technical note of the first successful single-incision laparoscopic total gastrectomy (SITG) with D1+beta lymph node dissection (LND) for a patient with early proximal gastric cancer.

\section{Case 1}

A 50 -year-old man with a body mass index of $19.5 \mathrm{~kg} / \mathrm{m}^{2}$ was diagnosed with a moderately differentiated early gastric adenocarcinoma by endoscopy as per national screening guidelines. Endoscopic ultrasonography indicated that the tumor invaded the submucosal layer of the high body of the posterior wall of the stomach. Computed tomography (CT) scan revealed that there was no regional lymph node (LN) enlargement or distant metastasis (cT1bNOM0). Informed consent for a single-incision laparoscopic total gastrectomy was obtained.

\section{Operative technique}

Single-incision laparoscopic total gastrectomy with D1+beta LN dissection

The patient was placed in a lithotomy position with reverse Trendelenburg. The operator and a scopist were positioned between the patient's legs. A longitudinal $2.5-\mathrm{cm}-\mathrm{long}$ trans-umbilical skin incision was made. A commercial four-hole single port (Glove port; Nelis, Bucheon-si, 
Gyeonggi-do, Korea) was then placed in the umbilical incision, and the abdominal cavity was insufflated with carbon dioxide at a pressure of $13 \mathrm{mmHg}$ (Fig. 1). There was no additional assistant trocar. A 10-mm flexible highdefinition scope (Endoeye flexible HD camera system; Olympus Medical Systems, Tokyo, Japan) and a Harmonic Scalpel (Ethicon Endo-Surgery, Cincinnati, OH, USA) were used to visualize every corner of the operative field and facilitate dissection. We used the conventional laparoscopic grasper when operating in the greater curvature side and the prototype curved instruments (Olympus Medical Systems) when operating in the lesser curvature side, including the suprapancreatic LND and division of the esophagus. Modified combined suture retraction of the falciform ligament and the left lobe of the liver was performed using 2-0 prolene on a straight needle and 5-mm hemoclips [8]. Partial omentectomy was initiated distally approximately $3-4 \mathrm{~cm}$ away from the gastroepiploic arcade, which included the LN 4d. To prevent omental infarction, the left gastroepiploic vessels were ligated distal
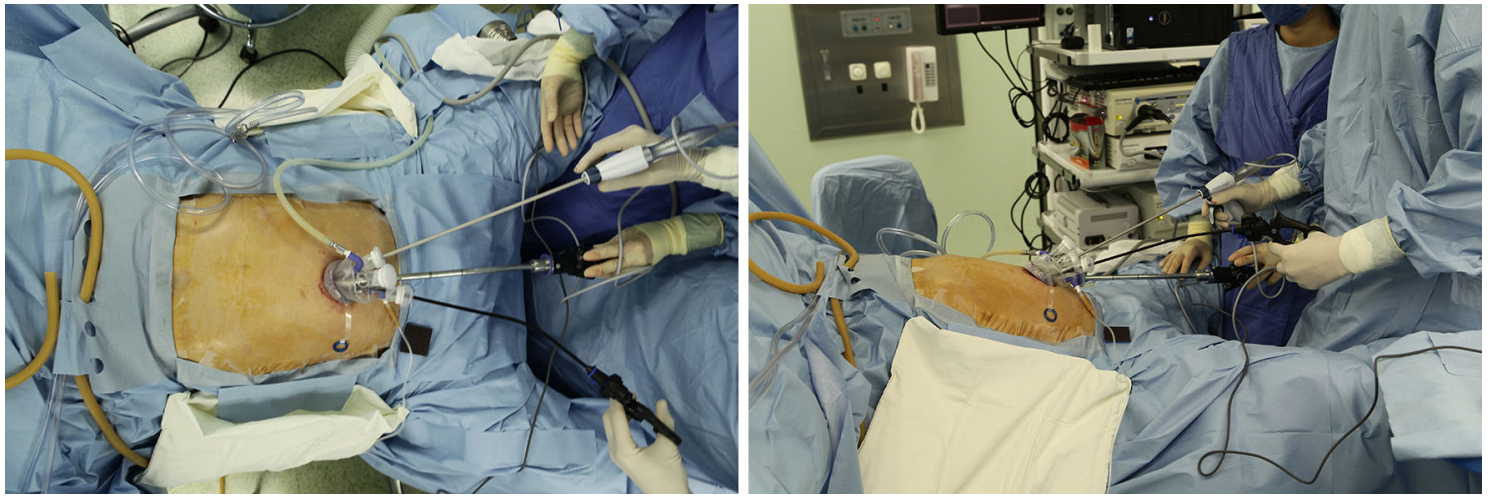

Fig. 1 Position and single-port placement
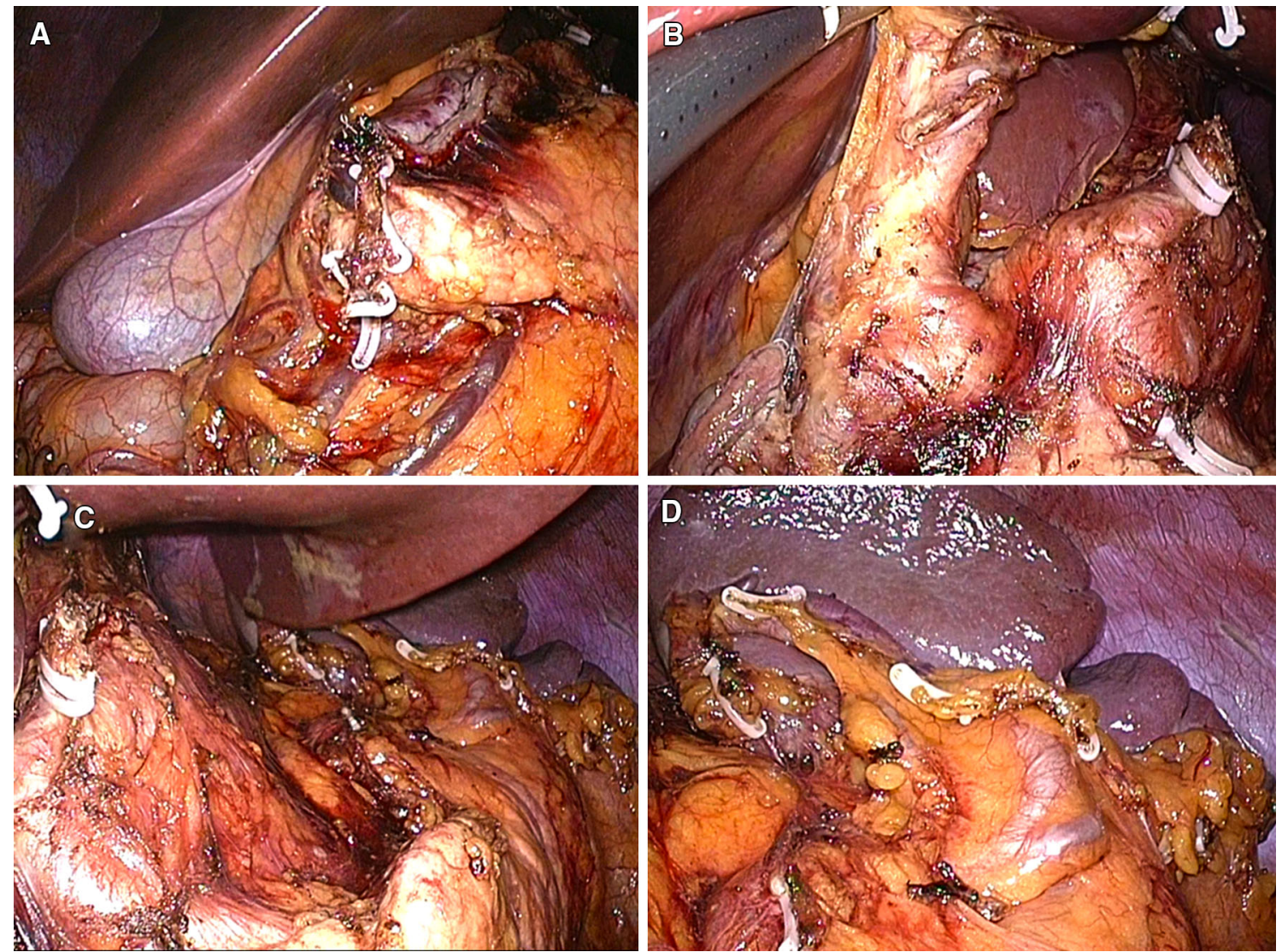

Fig. 2 Lymph node dissection in case 1: a LN \#6; b LN \#7, 8a, 12a, 7; c 7, 9, 11p; d LN \#10, 11d) 

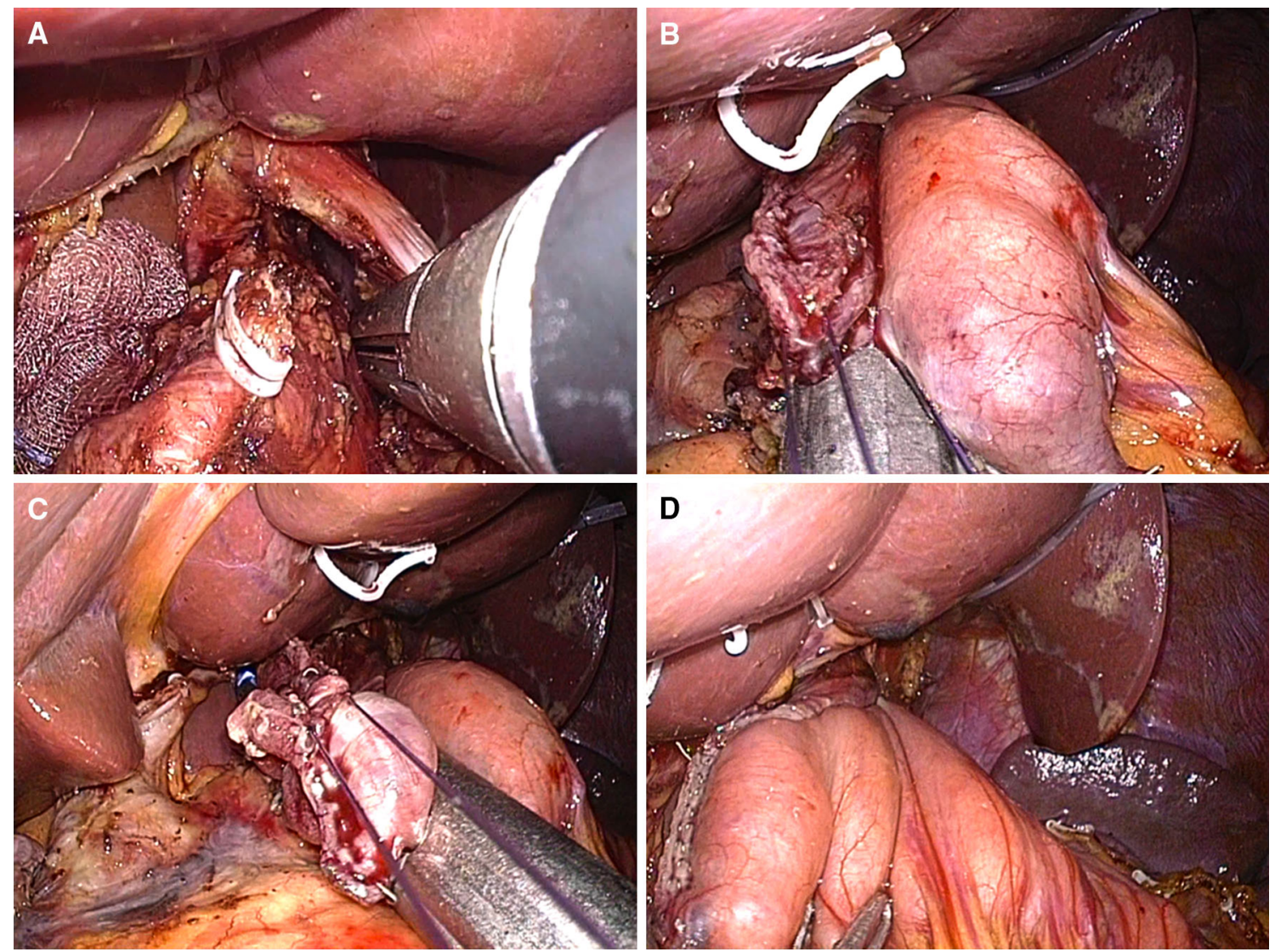

Fig. 3 Modified semi-loop esophagojejunostomy in case 1: esophagus transection (a), esophagojejunostomy (b), common entry hole closure by a linear stapler (c), complete view of modified semi-loop esophagojejunostomy (d)

to the omental branch. Then, the omentum was dissected and taken down from the mesocolon to the head of the pancreas and duodenum. The right gastroepiploic arcade was approached in a retrograde fashion. We first dissected the space between the duodenum and the basin including the right gastroepiploic vessels and LN station 6 and then detached these from the duodenum and distal stomach. Thus, we could easily dissect and divide the right gastroepiploic area without any significant bleeding. After dissecting LN 6, the right gastric artery and the proper hepatic artery were adequately exposed to dissect LNs 5 and 12a, and the operator exchanged the grasper for the prototype curved instruments. The right gastric artery was then divided at its origin. The duodenum was divided $2 \mathrm{~cm}$ distal to the pylorus using a laparoscopic linear stapler (Echelon $60 \mathrm{~mm}-3.5$ and 4.5; Ethicon). LNs 8a and 9, located on the right side of the left gastric artery, were dissected along each artery. The left gastric vein and artery were exposed, individually clipped, and divided to allow dissection of LN 11p. LNs 1 and 2, including the vagus nerve, were dissected and the esophagus was then transected using the stapler. The dissection subsequently proceeded along the LN stations 11p and 11d up to the splenic hilum. The dissection was carried out by the "downstream method" for LNs 2 and 4sa (Fig. 2). The specimen was removed in a plastic bag from the single umbilical incision without any extension.

\section{Reconstruction}

For the intracorporeal anastomosis, we used the modified semi-loop reconstruction for SITG [9]. The jejunal loop was transected approximately $20 \mathrm{~cm}$ distal to the ligament of Treitz, using the laparoscopic linear stapler without any division of the mesentery. After a small hole was made at the end of the Roux-en-Y limb on the antimesenteric side and on the left side of the esophageal stump, one stay sutures were placed at the esophageal stump to maintain traction through the single port and another stay sutures were placed at the small hole to facilitate insertion of the stapler. Through the blue channel of the single port, the stapler was inserted. The stapler side was inserted into the small hole of the jejunum while the anvil side was inserted into the small hole of esophageal stump using traction from the stay sutures. After firing the stapler, we carefully checked the lumen to identify any false lumen formation and luminal bleeding. The common entry was simply closed using the stapler with some 

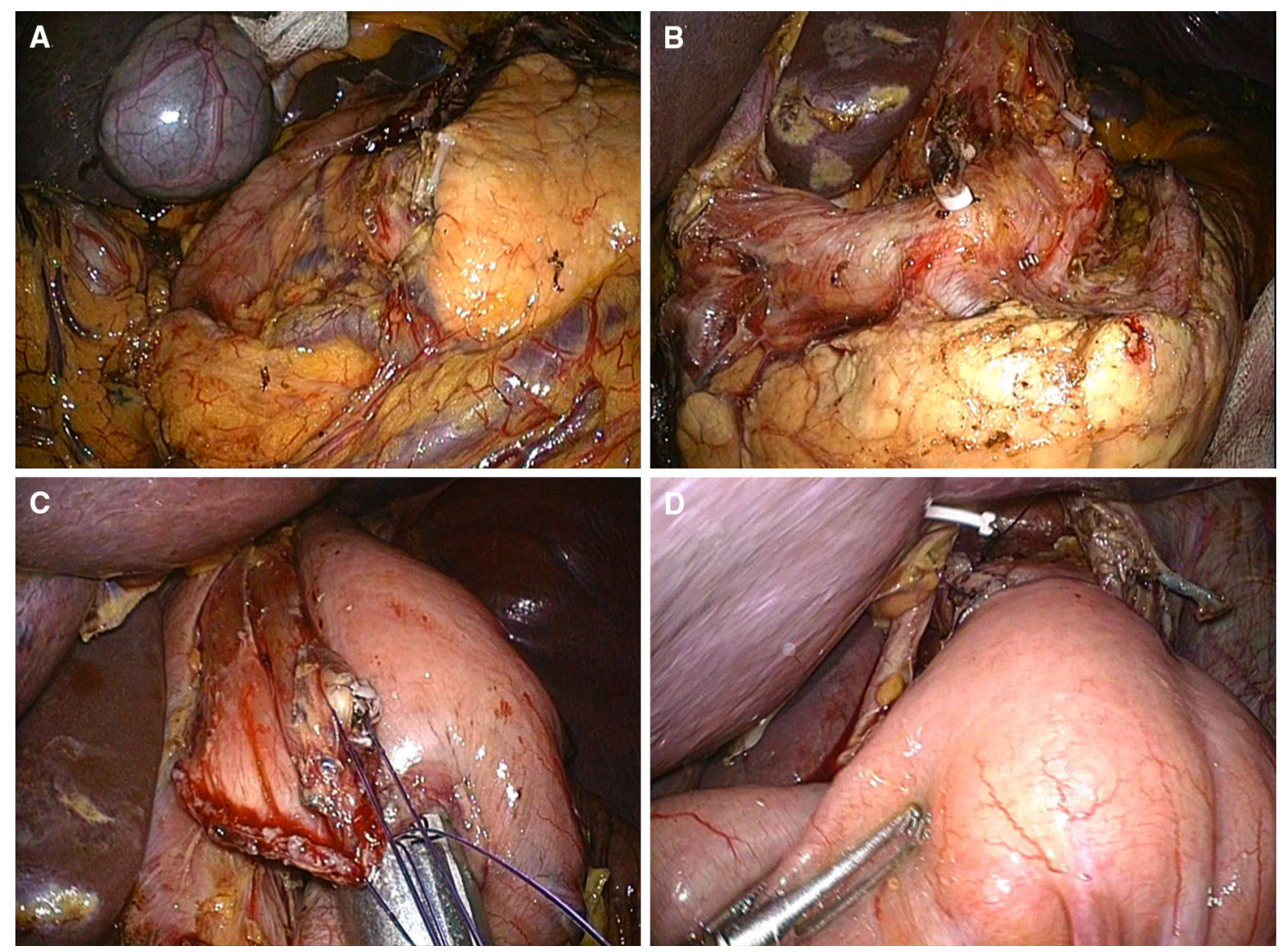

Fig. 4 Lymph node dissection and esophagojejunostomy in case 2: a LN \#6; b suprapancreatic LN area, 8a, 9, 7, 11p; c esophagojejunostomy by a linear stapler; $\mathbf{d}$ complete view of modified semi-loop esophagojejunostomy

staying suture, incorporating both the esophageal and jejunal stumps (Fig. 3). Side-to-side jejunostomy was then performed using the linear stapler.

\section{Postoperative outcomes}

The total operative time was $175 \mathrm{~min}$. Estimated blood loss was $50 \mathrm{ml}$. The patient was started on clear fluids on postoperative day 3 and tolerated a soft bland diet on postoperative day 4. Routine patient-controlled analgesia was administered for postoperative pain control, with good effect. The patient was discharged in stable condition without any complications or complaints on postoperative day 6 . Results of final pathological analysis revealed no LN metastasis among 56 examined nodes (pT1bN0). The proximal and distal resection margin was 2.1 and $9.8 \mathrm{~cm}$, respectively.

\section{Case 2}

The other case was a 46-year-old man diagnosed as having a poorly cohesive gastric carcinoma. Endoscopic ultrasonography and computed tomography revealed the preoperative tumor stage to be cT1bN0M0. He had no severe comorbidity. His body mass index was $18.4 \mathrm{~kg} / \mathrm{m}^{2}$. $\mathrm{He}$ also underwent SITG with D1+beta LN dissection in the same manner as already described (Fig. 4). The operation time was $205 \mathrm{~min}$, and the estimated amount of blood loss was $60 \mathrm{ml}$. He started sips of water on the postoperative 3rd day and ate a semibland diet on the postoperative 5th day. He was discharged with no complication and complaints on the postoperative 6th day. The final pathology report revealed that the tumor diameter was $7.2 \mathrm{~cm}$ and had invaded the muscularis propria; there were two metastases among 97 LNs dissected (pT2N1). The proximal and distal resection margins were 2 and $11.7 \mathrm{~cm}$, respectively.

\section{Discussion}

Single-incision laparoscopic surgery was introduced to reduce the minimal invasiveness of laparoscopy to the least invasiveness possible and to achieve excellent cosmetic results. SILS has been applied in various operations, with predominantly benign indications.

SILS is rarely applied for gastric cancer surgery. To the best of our knowledge, there have been only three reports 


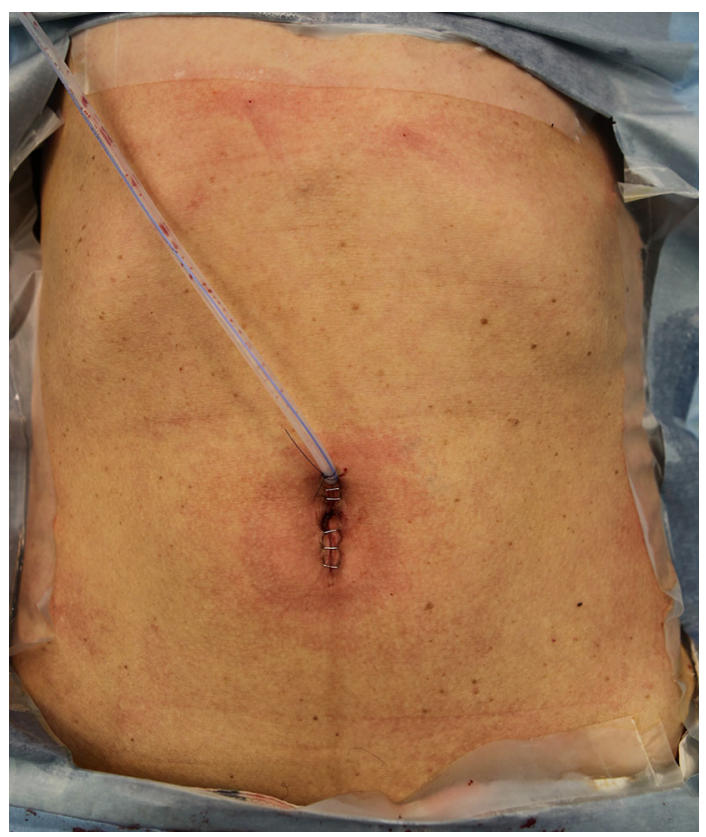

Fig. 5 Single-incision wound after the operation

describing this procedure for patients with EGC [5-7]. Furthermore, all these reports involve SIDG with one or two additional assistant ports.

This is the first case report describing successful singleincision laparoscopic total gastrectomy for early gastric cancer and also the first "pure" SITG without any additional assistant port. In our institution, we conducted an animal study in porcine models before the clinical application of SILS for EGC. We found that D1+beta LND could be performed during SIDG in this animal model [10].

After this animal study, we performed SIDG in 11 EGC patients with the approval of our institutional review board. We reported the first two cases as case reports [6]. Based on these experiences, we prepared to perform pure SITG. The indication of SITG and conventional laparoscopic total gastrectomy (LTG) in our institution was clinical stage I (cT1-T2N0). The initial decision to perform SITG or LTG was mainly determined by the patient's body mass index, which was between 18 and $21 \mathrm{~kg} / \mathrm{m}^{2}$.

For pure SITG, the single-port device is very important for simple and less stressful surgery. The Glove port (Nelis, Buchen, Gyeonggi, Korea) is very effective, with the angle easily adjustable and no air leakage. Each valve can use 2- to $12-\mathrm{mm}$ instruments. In addition, because the port is transparent, instruments can be inserted under direct visualization.

However, it can be difficult to maintain an optimal view in SITG, because of repeated clashes of the camera with the instruments. This technical challenge can be overcome with a flexible HD camera and an experienced camera operator. Further, a curved grasper can improve surgical performance, with less interference, thereby facilitating
SILS with reduced time required and improved dexterity. In the future, a 5-mm flexible HD scope may help resolve the clashing of instruments, as more space would be available to move the instruments.

Compared with other laparoscopic approaches, the postoperative cosmetic result from this operation was excellent (Fig. 5), and the incisional pain was minimal on postoperative day 1 . The total operative time was similar to that of conventional laparoscopic total gastrectomy. Therefore, this technical report demonstrated the technical possibility of pure SITG and modified semi-loop esophagojejunostomy. Further experience and research are required to confirm the safety and feasibility of SITG.

Conflict of interest Drs. S.-H. Ahn, D.J. Park, S.Y. Son, C.-M. Lee, and H.-H. Kim have no conflicts of interest or financial ties to disclose.

\section{References}

1. Kim HH, Hyung WJ, Cho GS, Kim MC, Han SU, Kim W, Ryu SW, Lee HJ, Song KY. Morbidity and mortality of laparoscopic gastrectomy versus open gastrectomy for gastric cancer: an interim report-a phase III multicenter, prospective, randomized trial (KLASS Trial). Ann Surg. 2010;251(3):417-20.

2. Hwang SH, Park do J, Jee YS, Kim MC, Kim HH, Lee HJ, Yang HK, Lee KU. Actual 3-year survival after laparoscopy-assisted gastrectomy for gastric cancer. Arch Surg 2009;144(6):559-564; discussion 565

3. Bucher P, Pugin F, Morel P. Single-port access laparoscopic radical left colectomy in humans. Dis Colon Rectum. 2009;52(10):1797-801.

4. Reavis KM, Hinojosa MW, Smith BR, Nguyen NT. Single-laparoscopic incision transabdominal surgery sleeve gastrectomy. Obes Surg. 2008;18(11):1492-4.

5. Omori T, Oyama T, Akamatsu H, Tori M, Ueshima S, Nishida T. Transumbilical single-incision laparoscopic distal gastrectomy for early gastric cancer. Surg Endosc. 2011;25(7):2400-4.

6. Park do J, Lee JH, Ahn SH, Eng AK, Kim HH. Single-port laparoscopic distal gastrectomy with $\mathrm{D} 1+$ beta lymph node dissection for gastric cancers: report of 2 cases. Surg Laparosc Endosc Percutan Tech 2012;22(4):e214-216.

7. Omori T, Tanaka K, Tori M, Ueshima S, Akamatsu H, Nishida T. Intracorporeal circular-stapled Billroth I anastomosis in singleincision laparoscopic distal gastrectomy. Surg Endosc. 2012;26(5):1490-4.

8. Shabbir A, Lee JH, Lee MS, Park do J, Kim HH. Combined suture retraction of the falciform ligament and the left lobe of the liver during laparoscopic total gastrectomy. Surg Endosc 2010;24(12):3237-3240.

9. Okabe H, Obama K, Tanaka E, Nomura A, Kawamura J, Nagayama S, Itami A, Watanabe G, Kanaya S, Sakai Y. Intracorporeal esophagojejunal anastomosis after laparoscopic total gastrectomy for patients with gastric cancer. Surg Endosc. 2009;23(9):2167-71.

10. Lee JH, Lee MS, Kim HH, Park do J, Lee HJ, Yang HK, Park KU. Comparison of single-incision laparoscopic distal gastrectomy and laparoscopic distal gastrectomy for gastric cancer in a porcine model. J Laparoendosc Adv Surg Tech A 2011;21(10):935-940. 